\title{
Supersolidity of lattice Bosons immersed in strongly correlated Rydberg dressed atoms
}

\author{
Yongqiang Li, ${ }^{1}$ Andreas Geißler, ${ }^{2}$ Walter Hofstetter, ${ }^{2}$ and Weibin $\mathrm{Li}^{3,4}$ \\ ${ }^{1}$ Department of Physics, National University of Defense Technology, Changsha 410073, P. R. China \\ ${ }^{2}$ Institut für Theoretische Physik, Goethe-Universität, 60438 Frankfurt/Main, Germany \\ ${ }^{3}$ School of Physics and Astronomy, University of Nottingham, Nottingham NG7 2RD, UK \\ ${ }^{4}$ Centre for the Mathematics and Theoretical Physics of Quantum Non-equilibrium Systems, \\ University of Nottingham, Nottingham NG7 2RD, UK
}

(Dated: January 13, 2018)

\begin{abstract}
Recent experiments have illustrated that long range two-body interactions can be induced by laser coupling atoms to highly excited Rydberg states. Stimulated by this achievement, we study supersolidity of lattice bosons in an experimentally relevant situation. In our setup, we consider two-component atoms on a square lattice, where one species is weakly dressed to an electronically high-lying (Rydberg) state, generating a tunable, soft-core shape long-range interaction. Interactions between atoms of the second species and between the two species are characterized by local interand intra-species interactions. Using a dynamical mean-field calculation, we find that interspecies onsite interactions can stabilize a pronounced region of supersolid phases. This is characterized by two distinctive types of supersolids, where the bare species forms supersolid phases that are immersed in strongly correlated quantum phases, i.e. a crystalline solid or supersolid of the dressed atoms. We show that the interspecies interaction leads to a roton-like instability in the bare species and therefore is crucially important to the supersolid formation. We provide a detailed calculation of the interaction potential to show how our results can be explored under current experimental conditions.
\end{abstract}

\section{INTRODUCTION}

A supersolid is a translational symmetry breaking superfluid occurring in a solid. It was predicted to exist in bulk helium over forty years ago [1], but its observation has remained a challenge [2]. To reach supersolidity, one typically relies on long-range two-body interactions to break the translational invariance of a homogeneous system. Recent experiments have observed supersolid orders where translational symmetry is broken by cavity photon assisted [3] or spin-orbit coupling enabled [4] momentum transfer. To achieve supersolids induced purely by twobody interactions, enormous efforts have been spent on polar molecules [5, 6], magnetic [7] and Rydberg atom$\mathrm{s}[8,9]$, due to the available long-range atom-atom interaction as well as high precision control over their internal and motional states. However, a current challenge is that theoretical proposals typically examine regimes that are difficult to achieve experimentally.

In this work, we study supersolids of a two-species bosonic mixture on a two-dimensional (2D) square lattice, where one of the species is weakly coupled to an electronically high-lying (Rydberg) state by an off-resonant laser (the level scheme is depicted in Fig. 1a). Uniquely, this setting is recently realized experimentally at $\mathrm{Mu}-$ nich [10] in the study of Rydberg dressed spin dynamic$\mathrm{s}$ [11]. The coupling laser induces strong and long-range interactions between Rydberg dressed atoms on distances well beyond typical lattice-site spacings (see Fig. 1b), whose strength and sign can be controlled by the laser (i.e. detuning and Rabi frequencies) and the choices of Rydberg states [12]. The resulting Bose-Hubbard model features a long-range interaction between dressed atom-

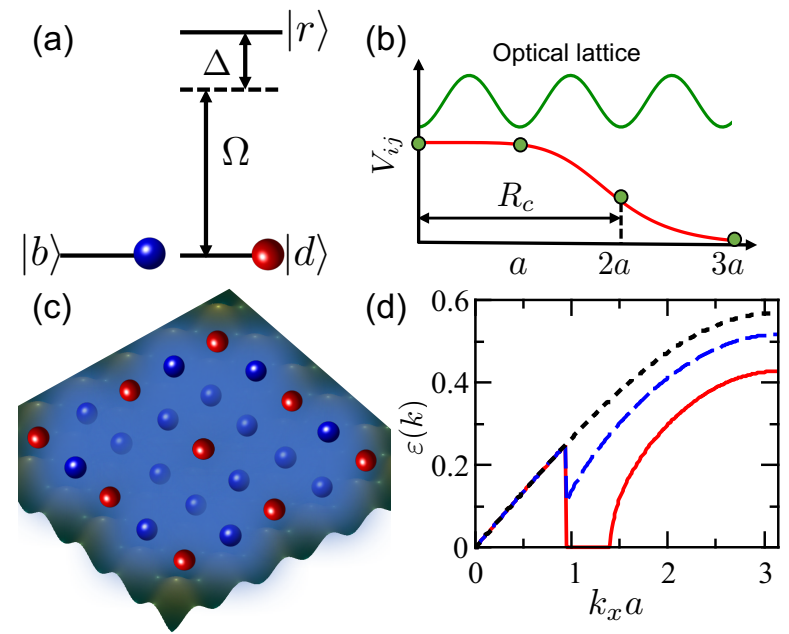

FIG. 1. (Color online) (a) Two electronic ground states $|b\rangle$ (blue) and $|d\rangle$ (red) and a Rydberg state $|r\rangle$ are considered. An off-resonant laser (with Rabi frequency $\Omega$ and detuning $\Delta$ ) weakly couples the state $|d\rangle$ to $|r\rangle$. (b) The soft-core shape interaction potential $V_{i j}$ (red) between atoms in the Rydberg dressed state $|d\rangle$. The soft-core radius $R_{c}$ can be larger than the lattice spacing $a$. Here $R_{c}=2 a$ is shown. (c) SS of the bare state when dressed atoms are in an ordered density wave (DW). (d) Roton instability of the bare species. The Bogoliubov dispersion relation (along the $k_{x}$ axis) of phonons is significantly modified by the interspecies interaction. A rotonlike instability emerges when the interspecies interaction $U_{b d}$ is increased, indicating that the ground state phase changes from a homogeneous superfluid to supersolid. In the figure we show $U_{b d} / U=0$ (dotted), $U_{b d} / U=0.45$ (dashed) and $U_{b d} / U=1$ (solid). Other parameters are $k_{y}=0, V / U=0.4$ and $t / U=0.04$. See text for details. 
$\mathrm{s}$ while interactions between atoms of the two different species and of the bare species are short ranged.

Employing real-space bosonic dynamical mean-field theory (RBDMFT), we find that the system undergoes a series of many-body phases, including Mott insulator (MI), ordered density-wave (DW), supersolid (SS) and superfluid (SF) phases. A key result is that the interspecies interaction enables supersolid phases of the bare species in regions where the dressed atoms are in DW or SS phases (an example for a DW is depicted in Fig. 1c). Using Bogoliubov theory, we reveal that a roton-like instability emerges due to the interspecies interaction (see Fig. 1d), which signifies a SF to SS transition [13]. Our results open a new route to enhance the formation of SS phases through the Rydberg dressing in two-component atomic gases.

The paper is organised as follows. In Sec. II we present the two-component Bose-Hubbard model of the system, in which the dressed atoms interact via a long-range softcore shape interaction. In Sec. III, ground state phase diagrams of the model are systematically examined at low particle fillings using the RBDMFT. A key finding is that the species with short range interactions exhibits a supersolid state. In Sec. IV, the supersolid mechanism of the bare species is studied, where a roton instability is found due to interstate interactions. In Sec. V, we discuss how one can engineer the required long-range interactions and explore the interesting phases in cold atom experiments. We conclude in Sec. VI.

\section{THE HAMILTONIAN OF THE SYSTEM}

In sufficiently deep lattices, our setting is described by a single band, two-component Bose-Hubbard model,

$$
\hat{H}=-\sum_{\langle i j\rangle, \sigma} t_{\sigma}\left(\hat{b}_{i \sigma}^{\dagger} \hat{b}_{j \sigma}+\text { H.c. }\right)+\sum_{i<j} V_{i j} \hat{n}_{i d} \hat{n}_{j d}-\sum_{i} \hat{H}_{i},
$$

where the single site Hamiltonian $\hat{H}_{i}=$ $\frac{1}{2} \sum_{\sigma \sigma^{\prime}} U_{\sigma \sigma^{\prime}} \hat{n}_{i \sigma}\left(\hat{n}_{i \sigma^{\prime}}-\delta_{\sigma \sigma^{\prime}}\right)-\sum_{\sigma} \mu_{\sigma} \hat{n}_{i \sigma} . \quad\langle i, j\rangle$ represents the nearest neighbour sites $i, j$. Index $\sigma\left(\sigma^{\prime}\right)=b, d$ denotes bare, and dressed states, respectively. $\hat{b}_{i \sigma}^{\dagger}\left(\hat{b}_{i \sigma}\right)$ and $\hat{n}_{i \nu}=\hat{b}_{i \nu}^{\dagger} \hat{b}_{i \nu}$ are the bosonic creation (annihilation) operator for species $\sigma$ and atomic density at site $i$. $t$ and $\mu_{\sigma}$ determine the hopping rate and chemical potential for the two bosonic species. We assume the intra-species interactions $U_{b, d}$ are identical for both species [14].

In this model, we consider both short-range and long-range interactions. $U_{\sigma \sigma^{\prime}}$ denotes the inter- and intra-species short-range (onsite) interactions, which can be tuned via e.g. Feshbach resonances [15] or statedependent optical lattices [16]. The long-range interaction between site $i$ and $j$ is $V_{i j} \equiv V /\left[\left(a / R_{c}\right)^{6}(i-j)^{6}+1\right]$, where $V=\tilde{C}_{6} / R_{c}^{6}$ characterises the long-range interaction at a distance $R_{c}$. $\tilde{C}_{6}, R_{c}$ and $a$ are the effective dispersion coefficient, soft-core radius, and lattice constant, respectively. In the following, we choose the intraspecies short-range interaction $U_{b, d} \equiv U$, which also sets the unit of energy. Details of these parameters will be given in Section V.

\section{MANY-BODY GROUND STATE PHASE DIAGRAM}

In this section, we study the stability of quantum phases of Rydbery-dressed systems in optical lattices for fillings $0 \leq\left\langle n_{i}\right\rangle \leq 2$. To determine the ground state phases, we use RBDMFT to capture both higher order quantum fluctuations, strong correlations and arbitrary long-range order in a unified framework $[17,18]$. It provides a nonperturbative description of many-body systems in two and three spatial dimensions (the method is discussed in Appendix A.). In the calculations, we typically consider the lattice size as large as $N_{\text {lat }}=48 \times 48$ sites and an experimentally relevant soft-core radius $R_{c}=3 a$ [12]. The superfluidity is characterised by the condensate order parameter $\phi_{\sigma} \equiv\left\langle\hat{b}_{\sigma}\right\rangle$, and crystalline order by the realspace density distribution $n_{i \sigma}=\left\langle\hat{n}_{i \sigma}\right\rangle$ and total density $n_{i} \equiv n_{i b}+n_{i d}$. The coexistence of both condensate and crystalline order parameters gives the supersolid phase.

\section{A. Density dependent phase diagram of Rydberg-dressed systems}

In this section, we study features of the phase diagram at various fillings. In the strong coupling limit with $U_{\sigma \sigma^{\prime}} \gg t$, we find that the system favors Mott insulating or density-wave phase with different types of crystalline order in the individual species. Interestingly, we observe a density-wave phase with a nonuniform total density which breaks lattice translational symmetry, with densities $n_{i b}=1$ and $n_{i d}=2$, appears, as shown in green region of Fig. 2. These density-waves exhibit nonzero density fluctuations, as shown in Fig. 3. However, quantum fluctuations as a result of higher-order tunneling processes are weak, due to the strong long-range interactions. Actually, the density-wave of the dressed species is also predicted in the single-species case [8].

Away from the deep MI regime, i.e. in the intermediate hopping regime, we observe two types of quantum phase transition from MI to supersolid, i.e. the uncoupled ground-state species demonstrates a phase transition from MI to a supersolid (SS1) formed by the bare species while the dressed atom is in a MI. Further increasing the tunnelling rate $t$, both species are in a supersolid state (SS2), as shown in the Fig. 2. Interestingly, we observe a pronounced region of supersolid appearing in our simulations, as a result of the onsite interspecies interactions, indicating a higher chance for directly observing these phases in realistic experiments, compared to singlespecies case [8]. Actually, we indeed observe the width of SS1 and SS2 shrinks as a function of interspecies interactions, as shown in Fig. 2(f), where SS1 clearly disappears 


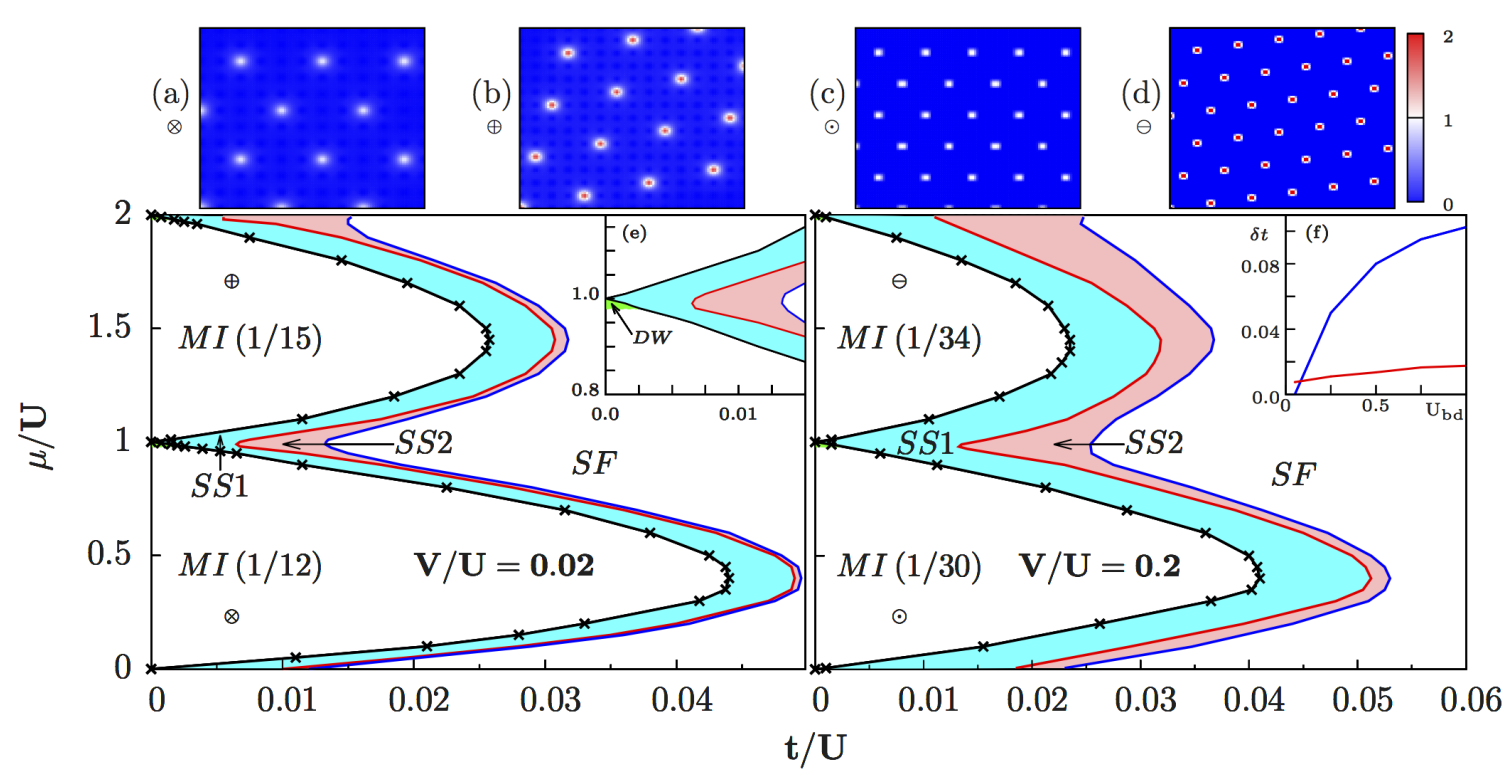

FIG. 2. (Color online) Phase diagram on a square lattice for Rydberg dressed interaction $V / U=0.02$ and 0.2 , respectively, demonstrating stable supersolid regions marked by the cyan (SS1) and pink color (SS2). In the Mott-insulating phase (MI) with spatially uniform total density, the Rydberg dressed species exhibits different crystalline order, as shown in (a)-(d) for real-space density $n_{d}$, with lattice sizes being the square of the area of the unit cell of the Rydberg dressed species $\left[N_{\text {lat }}=12 \times 12(\otimes)\right.$; $N_{\text {lat }}=15 \times 15(\oplus) ; N_{\text {lat }}=30 \times 30(\odot)$; and $\left.N_{\text {lat }}=34 \times 34(\ominus)\right]$. Inset: density-wave phase $($ DW $)$ with density $n_{b}=1$ for the ground-state species and $n_{d}=2$ for the Rydberg dressed state in the corresponding filled sites, respectively (e), and width of supersolid phase [SS1 (blue) and SS2 (red)] $\delta t \equiv t_{\mathrm{c} 1}-t_{\mathrm{c} 2}$ as a function of interspecies interaction $U_{b d} / U$ for Rydberg dressed interaction $V / U=0.2$ and chemical potential $\mu / U=0.4$ (f). Other parameters are $U_{b d}=U, \mu / U=\mu_{b} / U=\mu_{d} / U-0.05$.

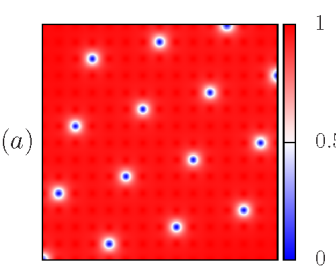

$n_{b}$

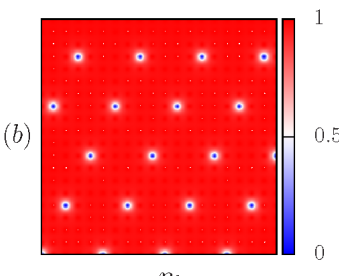

$n_{b}$

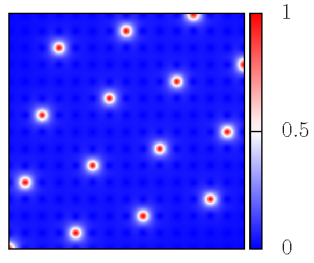

$n_{d}$

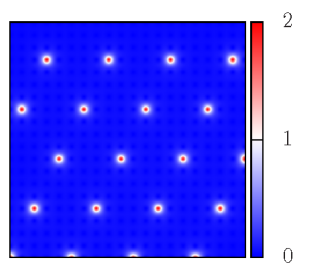

$n_{d}$

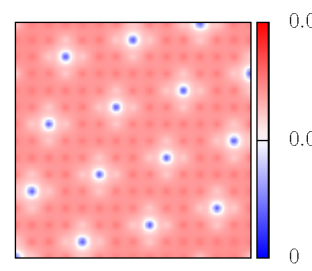

$\Delta n_{b}$

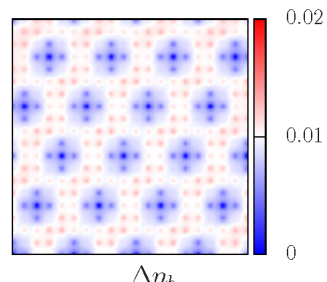

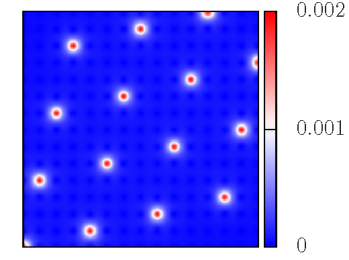

$\Delta n_{d}$

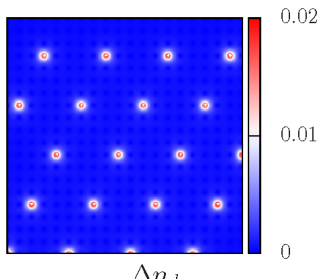

FIG. 3. (Color online) Real-space density $n_{b, d}$ and density fluctuations $\Delta_{b, d} \equiv\left(n_{b, d}-\left\langle n_{b, d}\right\rangle\right)^{2}$ in MI (a) and DW (b) phases, with lattice sizes being the square of the area of the unit cell of the Rydberg dressed species $\left[N_{\text {lat }}=15 \times 15\right.$ (a) $]$ and $\left[N_{\text {lat }}=20 \times 20\right.$ (b)], respectively. Other parameters are $t / U=0.03, V / U=0.3, U_{b d}=U, \mu_{b} / U=0.2$ and $\mu_{d} / U=0.7$ (a), and $t / U=0.0023$, $V / U=0.02, U_{b d}=U, \mu_{b} / U=0.98$ and $\mu_{d} / U=1.03(\mathrm{~b})$.

for smaller $U_{b d}$. In addition, the long-range interaction also shifts the phase transition between MI and SS1, even though the bare species only possess onsite interactions.

Finally, in the weakly interacting regime with $t \gg$ $U_{\sigma \sigma^{\prime}}$, a superfluid phase with uniform total density distribution is found in our simulations, where both species demonstrate homogenous density distribution. Here, crystalline orders are destroyed by the large density fluctuations, and the system only supports superfluidity with uniform density.

Note that a similar model using dipolar gases has been numerically investigated using a mean-field Gutzwiller approach and by considering only the nearest-neighbor part of the dipolar interactions [19]. In our calculations, we take into account the whole range of the interaction potential. As a comparison the phase diagram of dipolar systems is given in Appendix B. 


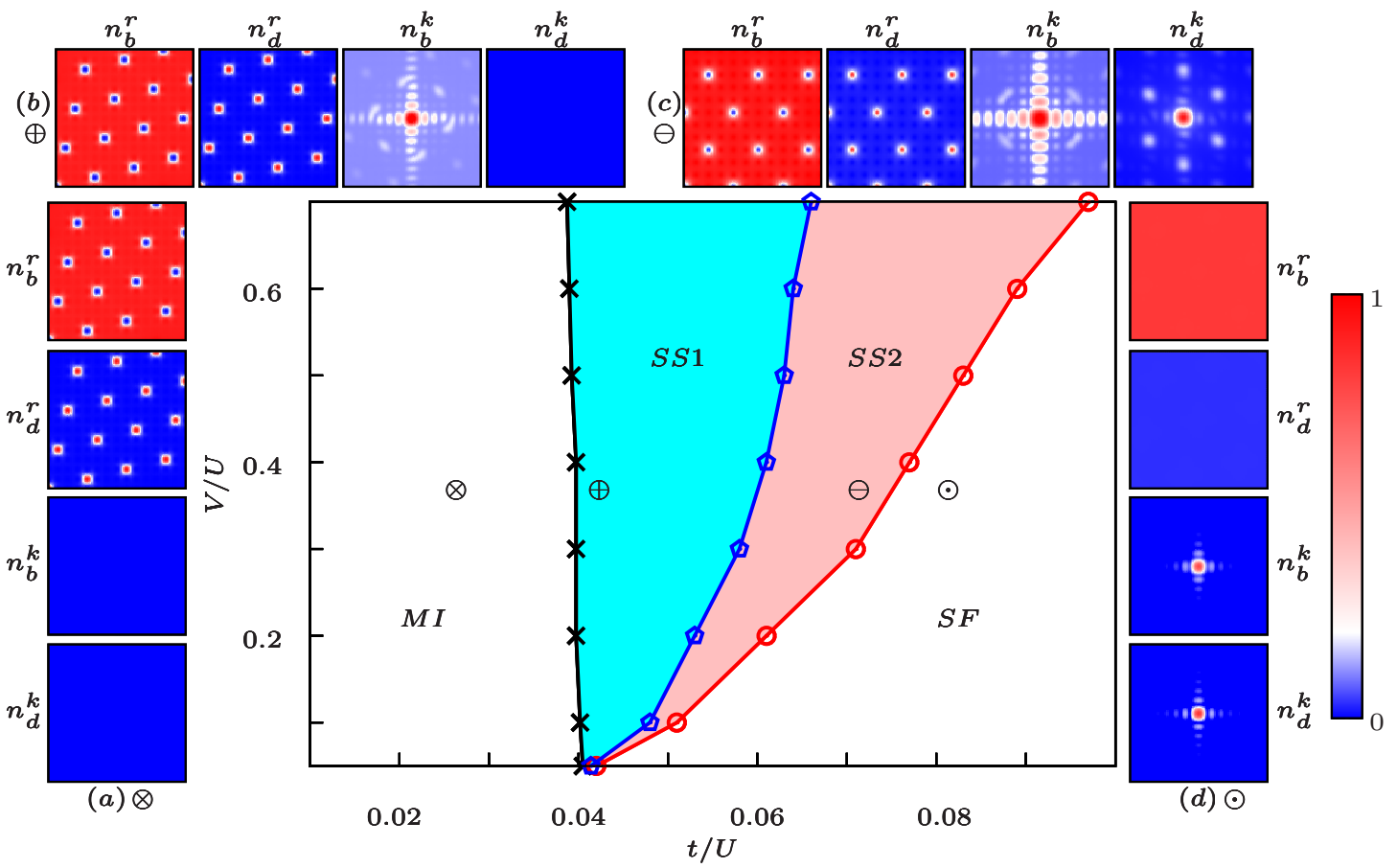

FIG. 4. (Color online) Phase diagram of a mixture of ground-state component $b$ and Rydberg dressed species $d$ on a square lattice in terms of hopping amplitude $t$ and Rydberg dressed interaction strength $V$. There are four stable phases in the diagram: Mott insulator (MI) with spatially uniform total local density and crystalline density order for each species, homogeneous superfluid (SF), and two types of supersolids (SS1 with Rydberg dressed species being in the crystalline phase, and SS2 with both species being in the supersolid). Other parameters are $U_{b d}=U$ and $n_{b}^{r}+n_{d}^{r}=1$. (a)-(d): Real-space density $n_{b, d}^{r}$ and quasi-momentum-space density $n_{b, d}^{k}$ distributions of different phases, with lattice sizes being the square of the area of the unit cell of the Rydberg dressed species $\left[\mathrm{MI}, N_{\text {lat }}=15 \times 15(\otimes) ; \mathrm{SS} 1, N_{\text {lat }}=15 \times 15(\oplus)\right.$; SS2, $N_{\text {lat }}=12 \times 12(\ominus)$; and $\left.\mathrm{SF}, N_{\text {lat }}=24 \times 24(\odot)\right]$, as shown by the markers in the main figure.

\section{B. Supersolid phases at unit filling}

In the following, we will focus on phases at unit filling $n_{j d}+n_{j b}=1$. We start with the so-called strong coupling limit when $U_{\sigma \sigma^{\prime}} \gg t$, where the $2 \mathrm{D}$ system favours MI phases with uniform total particle densities. Crystalline orders in the MI region can be changed by varying the two-body interactions (i.e. $V / U)$. One example is depicted in Fig. 4a, which shows relative densities and crystalline structures. Furthermore, when one increases $V / U$ continuously, the filling fractions $f_{d} \equiv \sum_{i} n_{i d} / N_{\text {lat }}$ of the dressed species can form a devil's staircase structure (Fig. 5a). An open question here is whether the staircase in this $2 \mathrm{D}$ system is complete. In $1 \mathrm{D}$ lattice systems, the devil's staircase and its completeness [20] have been extensively studied [21]. Moreover, there are very small regions occupied by DW phases (with a nonuniform total density).

When the hopping rate increases at fixed interaction $V / U$, we observe a pronounced region of supersolids. The bare state first enters the supersolid phase (SS1) from an insulating phase, while the dressed species is still crystallized in this case (one example is depicted in Fig. 4b).
Further increasing $t$, both species are in supersolid phases (SS2), as shown in Fig. 4c, where non-zero peaks appear for both species in addition to zero-momentum condensate, indicating the coexistence of non-trivial diagonal long-range order and off-diagonal long-range order associated with phase coherence. A large supersolid region indicates a higher chance for directly observing these phases in realistic experiments, compared to the single-species case [8].

One typically would not expect such supersolids as the bare species alone can only form superfluid and MI phases due to the short range two-body interactions [22]. The underlying mechanism is that the flow of the bare species is suppressed by the crystalline distribution of the dressed species via the interspecies interaction. As a result, the widths of the SS1 and SS2 phases will strongly depend on the interspecies interaction $U_{\mathrm{bd}}$. The numerical result in Fig. 5b shows that indeed the two SS phases shrink as $U_{b d}$ decreases. The SS1 phase eventually disappears for sufficiently small $U_{\mathrm{bd}}$. 

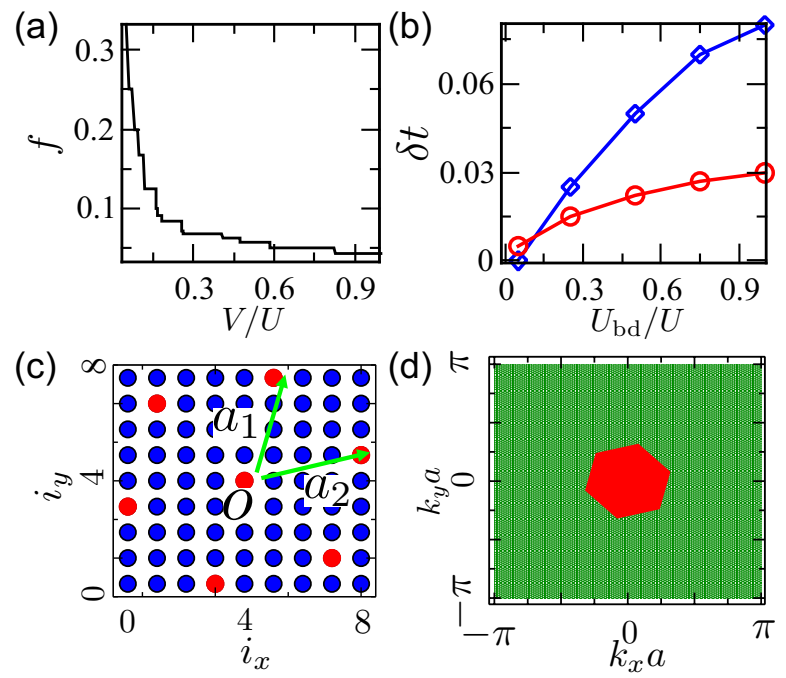

FIG. 5. (Color online) (a) Devil's staircase pattern of the filling fraction $f=\sum_{i} n_{i d} / N_{\text {lat }}$ for the Rydberg dressed species in the zero-hopping limit. (b) Width of supersolid phase SS1 (blue) and SS2 (red) $\delta t \equiv t_{\mathrm{c} 1}-t_{\mathrm{c} 2}$ as a function of interspecies interaction $U_{b d} / U$ for Rydberg dressed interaction $V / U=0.1$, where $t_{\mathrm{c} 1,2}$ denotes the critical value of the hopping amplitude of the upper/lower phase boundary of each phase shown in Fig. 4. (c) Density distribution of the dressed (red) and bare species (blue). The dressed atoms form an oblique lattice with lattice vector $a_{1}$ and $a_{2}$. This structure corresponds to the configuration illustrated in Fig. 4b. (d) The first Brillouin zone of the optical lattice (green) and oblique lattice (red) of the dressed atom. As the lattice vector $\left|a_{j}\right|>a(j=1,1)$, the size and shape of the first Brillouin zone of the dressed atoms differ significantly from the square reciprocal lattice of the optical lattice potential.

\section{SUPERSOLIDITY MECHANISM OF THE BARE SPECIES}

In the rest of the work, we will develop a Bogoliubov mean-field theory to understand how the interspecies interaction enables the bare species to form SS phases. Our discussion will focus on the SS1 phase, where the dressed species is a DW. This allows us to write down wave functions $\left|\mathrm{DW}_{d}\right\rangle$ of the DW according to the crystalline structure. We also assume that the total wave function in the ground state can be decoupled as $\left|\Psi_{\mathrm{g}}\right\rangle \approx\left|\mathrm{DW}_{d}\right\rangle \otimes\left|\Psi_{b}\right\rangle$, where $\left|\Psi_{b}\right\rangle$ is the wave function of the bare component. Then we can derive an effective Hamiltonian for the bare species by tracing out the dressed atom part, i.e. $\hat{H}_{\mathrm{e}}=\left\langle\mathrm{DW}_{d}|\hat{H}| \mathrm{DW}_{d}\right\rangle$. Explicitly the effective Hamiltonian reads,

$$
\begin{aligned}
\hat{H}_{\mathrm{e}}= & -\sum_{\langle i j\rangle} t\left(\hat{b}_{i}^{\dagger} \hat{b}_{j}+\text { H.c. }\right)+\frac{U}{2} \sum_{i} \hat{n}_{i}\left(\hat{n}_{i}-1\right) \\
& -\sum_{i} \mu \hat{n}_{i}+U_{b d} \sum_{\{j\}} \hat{n}_{j}
\end{aligned}
$$

where $\{j\}$ denotes lattice sites occupied by dressed atoms. For convenience, we have omitted the index $b$ of the bare species. The last term gives the interspecies interaction, where the mean particle number per site of the dressed atoms $n_{d}=1$ has been used explicitly. A constant term, $C=\left\langle\mathrm{DW}_{d}\left|\sum_{i<j} V_{i j} \hat{n}_{i d} \hat{n}_{j d}\right| \mathrm{DW}_{d}\right\rangle$ characterizing the long-range interaction energy, is neglected in the effective Hamiltonian.

The interaction with the dressed atoms (the last term in the effective Hamiltonian) introduces a new spatially periodic structure to the bare species, in addition to the optical lattice. As an example, we consider parameters corresponding to Fig. 4b. Here, the dressed atoms form an oblique lattice, see Fig. 5c for a cartoon picture of the $2 \mathrm{D}$ structure. The primitive cell of the new oblique lattice is apparently larger than the original lattice. In this example, the primitive lattice vectors are $a_{1}=(1,4)$ and $a_{2}=(4,1)$, with which we obtain the area of the primitive lattice $A=\left|a_{1} \times a_{2}\right|=15$, while the area of the optical lattice is 1 . In turn, the corresponding reciprocal lattice is smaller than that of the optical lattice. To illustrate this, we plot the first Brillouin zone of the two lattices in Fig. 5b. Apparently they overlap only in a small central area (low momentum regions).

As a result, phonon excitations for momentum components in and out of the overlap region will be very different. To show this, we calculate the Bogoliubov dispersion relation of the effective Hamiltonian (see details in Appendix C). In the low momentum region (where the two Brillouin zones overlap), $E_{k}^{\mathrm{i}}=$ $\sqrt{\varepsilon_{k}^{2}+2 \bar{n}_{b} U \varepsilon_{k}}$ with $\varepsilon_{k}=-2 t\left(\cos k_{x} a+\cos k_{y} a-2\right)$. Outside this region, the dispersion becomes $E_{k}^{o}=$ $\sqrt{\left(\varepsilon_{k}-\bar{n}_{d} U_{b d}\right)^{2}+2 \bar{n}_{b} U\left(\varepsilon_{k}-\bar{n}_{d} U_{b d}\right)}$. Here $\bar{n}_{b}\left(\bar{n}_{d}\right)$ are the mean population of the bare (dressed) component. Consequently, the dispersion is not continuous any more at the boundary of the Brillouin zone of the oblique lattice. The dispersion relation becomes complex when $U_{b d}>2 t \bar{n}_{d}\left(2-\cos k_{x}^{(b)} a-\cos k_{y}^{(b)} a\right)$ where $k_{x}^{(b)}$ and $k_{y}^{(b)}$ are momenta at the boundary. In Fig. 1d, we plot the dispersion relation along the $k_{x}$ axis by varying the interspecies interaction $U_{b d}$, where the mode frequency becomes complex at $U_{b d}=U$. This so-called roton-like instability [13] here indicates that the emergence of supersolids is indeed induced by the strong interspecies interaction. Note that the mechanism here is different from SS phases induced by geometrically dependent hopping found in frustrated lattices [23].

\section{LASER ENGINEERING OF THE DRESSED INTERACTIONS}

The level structure used in the Rydberg dressing is shown in Fig. 1a. The species $|d\rangle$ is coupled to a Rydberg state by an off-resonant laser with Rabi frequency $\Omega$ and detuning $\Delta$. Interactions between Rydberg atoms are of van der Waals type $V_{\mathrm{r}}=C_{6} / r^{6}$, where $C_{6}$ is the respective dispersion coefficient. The Rydberg dressing gives the soft-core interaction $V_{i j}$ where the effective dis- 


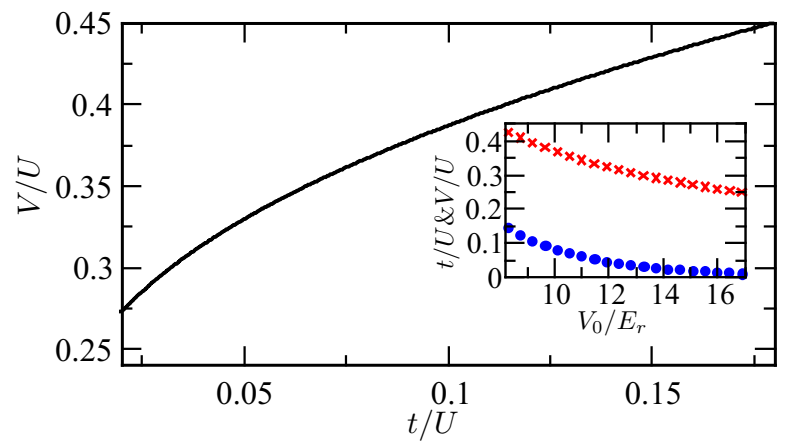

FIG. 6. (Color online) The hopping rate $t$ and onsite interaction $U$ depend on the lattice depth $V_{0} / E_{r}$. Increasing $V_{0} / E_{r}$, one can observe the phases discussed in the main text. The inset shows changes of $t / U(\bullet)$ and $V / U(\times)$ individually as a function of $V_{0} / E_{r}$. Here we consider the Rydberg state $36 \mathrm{~S}$ of ${ }^{87} \mathrm{Rb}$ atoms. Other parameters are $\lambda=1064 \mathrm{~nm}, a_{s}=5.2$ $\mathrm{nm}, C_{6}=241.6 \mathrm{MHz} / \mu \mathrm{m}^{6}, \Delta=7 \mathrm{MHz}$ and $\Omega=0.44 \mathrm{MHz}$.

persion coefficient $\tilde{C}_{6}=(\Omega / \Delta)^{4} C_{6}$ and soft-core radius $R_{c}=\left(C_{6} / 2 \Delta\right)^{1 / 6}$. $R_{c}$ varies with the Rydberg states and detuning. For example, one can choose the Rydberg $36 \mathrm{~S}$ state of ${ }^{87} \mathrm{Rb}$ atoms $\left(C_{6}=241.6 \mathrm{MHz} \times \mu m^{6}\right)$ and lattice constant $a=532 \mathrm{~nm}$. When $\Delta=7 \mathrm{MHz}$, we obtain $R_{c} \approx 3 a$. With this fixed detuning $\Delta$, the strength of the soft-core interaction is now controlled by the Rabi frequency $\Omega$.

To probe different phases shown in Fig. 4, one needs to change the parameters $V, U$ and $t$ together or separately over certain ranges. One simple way to achieve this is to tune the lattice potential depth $V_{0} / E_{r}$. In optical lattices, the onsite interaction $U$ depends on the lattice depth through $U=$ $\sqrt{8 / \pi} k a_{s} E_{r}\left(V_{0} / E_{r}\right)^{3 / 4}$ and the hopping rate $t$ through $t=4 / \sqrt{\pi} E_{r}\left(V_{0} / E_{r}\right)^{3 / 4} \exp \left[-2\left(V_{0} / E_{r}\right)^{1 / 2}\right]$ [24], where $k=2 \pi / \lambda, E_{r}=h^{2} / 2 m \lambda^{2}, \lambda=2 a$ and $a_{s}$ are the wave number, recoil energy, wavelength of the lattice potential and s-wave scattering length, respectively. Upon varying $V_{0} / E_{r}$ and fixing the other parameters, the ratios $t / U$ and $V / U$ change continuously. One example is shown in Fig. 6. One can see that the parameters cross the main phases discussed in this paper.

\section{CONCLUSIONS}

In conclusion, we have investigated crystalline phases of ultracold binary bosonic gases on a square lattice, with one species possessing a non-local interaction induced by Rydberg dressing. We found two types of supersolid phases that are robust and occupy large parameter regions at zero temperature. We showed that the supersolid phases of the bare species are stabilized by the interspecies interaction. The existence of the different phases predicted here could be directly observed by quantum gas microscopy with single-site resolution [25-27] or through measuring noise correlations [28]. Our results demonstrate rich features of the Bose-Bose mixture with long-range interactions, and indicate that this system is well suited for exploring supersolidity in upcoming experiments. As the crystalline structure (see Fig. 5a) can be changed in the insulating region by tuning $V / U$, we expect that supersolid phases with tunable density patterns can be explored as well. Moreover, physics in the SS2 region is not fully explored so far. It was shown that interesting phases, such as super-counter-fluid [29], can be created in a two-component Bose mixture. An open question here is whether new many-body phases can be expected when two supersolids coexist in the lattice system.

\section{ACKNOWLEDGMENTS}

We acknowledge J.-M. Yuan, Z.-X. Zhao and Rejish Nath for useful discussions. This work was supported by the National Natural Science Foundation of China under Grants No. 11304386 and No. 11104350. WL acknowledges support from the UKIERI-UGC Thematic Partnership No. IND/CONT/G/16-17/73 and EPSRC Grant No. EP/M014266/1. AG and WH acknowledge support from the Deutsche Forschungsgemeinschaft via DFG SPP 1929 GiRyd and the high-performance computing center LOEWE-CSC.
[1] A. F. Andreev and I. M. Lifshitz, Sov. Phys. JETP 29, 1107 (1969); G. V. Chester, Phys. Rev. A 2, 256 (1970); A J. Leggett, Phys. Rev. Lett. 25, 1543(1970).

[2] E. Kim and M. H. W. Chan, Nat. (London) 427, 225 (2004).

[3] K. Baumann et al., Nat. (London) 464, 1301 (2010); J. Léonard et al, Nat. (London) 543, 87 (2017).

[4] J. Li et al., Nat. (London) 543, 91 (2017).

[5] K.-K. Ni et al., Science 322, 231 (2008); S. A. Moses et al., Nat. (London) 350, 656 (2015); J. Deiglmayr et al., Phys. Rev. Lett 101, 133004 (2008); T. Takekoshi et al., Phys. Rev. Lett. 113, 205301 (2014); P. K. Molony et al., Phys. Rev. Lett. 113, 255301 (2014); J. W. Park et al.,
Phys. Rev. Lett. 114, 205302 (2015).

[6] B. Capogrosso-Sansone, C. Trefzger, M. Lewenstein, P. Zoller and G. Pupillo, Phys. Rev. Lett. 104, 125301 (2010); L. Pollet et al., Phys. Rev. Lett. 104, 125302 (2010).

[7] A. Griesma et al., Phys. Rev. Lett. 94, 160401 (2005); M. Lu et al., Phys. Rev. Lett. 107, 190401 (2011); K. Aikawa et al., Phys. Rev. Lett. 108, 210401 (2012).

[8] N. Henkel et al., Phys. Rev. Lett. 108, 265301 (2012); F. Cinti et al., Nat. Commun. 5, 3235 (2014); Marco Mattioli et al., Phys. Rev. Lett. 111, 165302 (2013); M. Dalmonte et al., Phys. Rev. B 92, 045106 (2015). 
[9] S. Baier et al., Science 352, 201 (2016); M. Iskin et al., Phys. Rev. A 79, 053634 (2009); A. Angelone, F. Mezzacapo, and G. Pupillo, Phys. Rev. Lett. 116, 135303 (2016).

[10] J. Zeiher et al, Nat. Phys. 12, 1095 (2016).

[11] A.W. Glaetzle et al., Phys. Rev. X 4, 041037 (2014); A. W. Glaetzle et al., Phys. Rev. Lett. 114, 173002 (2015); R.M.W. van Bijnen and T. Pohl, Phys. Rev. Lett. 114, 243002 (2015); Jan Gelhausen et al., arXiv:1608.01319; A. Geißler et al., Phys. Rev. A 95, 063608 (2017).

[12] I. Bouchoule and K. Mölmer Phys. Rev. A 65, 041803(R) (2002); N. Henkel, R. Nath and T. Pohl, Phys. Rev. Lett. 104, 195302 (2010); F. Maucher et al., Phys. Rev. Lett. 106, 170401 (2011); C. Gaul et al., arXiv:1511.06424; J. Honer et al., Phys. Rev. Lett. 105, 160404 (2010); W. Li, L. Hamadeh, and I. Lesanovsky, Phys. Rev. A 85, 053615 (2012); J. B. Balewski et al., New Journ. Phys. 16, 063012 (2014); J. E. Johnson and S. L. Rolston, Phys. Rev. A 82, 033412 (2010); G. Pupillo et al., Phys. Rev. Lett. 104, 223002 (2010); F. Cinti et al., Phys. Rev. Lett. 105, 135301 (2010); C. Gaul et al., Phys. Rev. Lett. 116, 243001 (2016); Y.-Y. Jau et al., Nat. Phys. 12, 71 (2016).

[13] L. Santos, G. V. Shlyapnikov, P. Zoller and M. Lewenstein, Phys. Rev. Lett. 85, 1791 (2000).

[14] S. Trotzky, P. Cheinet, S. Fölling, M. Field, U. Schnorrberger, A.M. Rey, A. Polovnikov, E.A. Demler, M.D. Lukin and I. Bloch, Science 319, 295 (2008); D.M. Weld, P. Medley, H. Miyake, D. Hucul, D. E. Pritchard, andW. Ketterle, Phys. Rev. Lett. 103, 245301 (2009).

[15] A. Widera, O. Mandel, M. Greiner, S. Kreim, T. W. Hänsch, and I. Bloch, Phys. Rev. Lett. 92, 160406 (2004)

[16] B. Gadway, D. Pertot, R. Reimann, and D. Schneble, Phys. Rev. Lett. 105, 045303 (2010).

[17] Y.-Q. Li et al., Phys. Rev. B 84, 144411 (2011); Phys. Rev. A 85, 023624 (2012); Phys. Rev. A 87, 051604(R) (2013); New Journ. Phys. 15, 093028 (2013).

[18] A. Georges et al., Rev. Mod. Phys. 68, 13 (1996); K. Byczuk et al., Phys. Rev. B 77, 235106 (2008); A. Hubener et al., ibid. 80, 245109 (2009); W. Hu et al., ibid. 80, 245110 (2009); P. Anders et al., Phys. Rev. Lett. 105, 096402 (2010); Y. Li et al., Phys. Rev. A 93, 033622 (2016).

[19] R. M. Wilson, W. E. Shirley, and S. S. Natu, Phys. Rev. A 93, 011605(R) (2016).

[20] P. Bak and R. Bruinsma, Phys. Rev. Lett. 49, 249 (1982).

[21] H. Weimer et al., Phys. Rev. Lett. 105, 230403 (2010); P. Hauke et al., New J. Phys. 12, 113037 (2010); F. J. Burnellet al., Phys. Rev. B 80, 174519 (2009); M. Bauer et al., Phys. Rev. Lett. 108, 255302 (2012); Z. Lan et al., Phys. Rev. Lett. 115, 203001 (2015).

[22] M. P. A. Fisher et al., Phys. Rev. B 40, 546 (1989); D. Jaksch et al., Phys. Rev. Lett. 81, 3108 (1998); M. Greiner et al., Nat. (London) 415, 39 (2002).

[23] T. Mishra, S. Greschner, and L. Santos, Phys. Rev. B 92, 195149 (2015).

[24] W. Zwerger, Journal of Optics B 5, 9 (2003).

[25] W. S. Bakr et al., Nat. (London) 462, 74 (2009).

[26] J. F. Sherson et al., Nat. (London) 467, 68 (2010).

[27] T. Gericke et al., Nat. Phys. 4, 949 (2009).

[28] V. W. Scarola, E. Demler, and S. Das Sarma, Phys. Rev. A 73, 051601(R) (2006).

[29] Anatoly Kuklov, Nikolay Prokofev, and Boris Svistunov, Phys. Rev. Lett. 92, 030403 (2004).

\section{Appendix A: The real-space bosonic dynamical mean-field method}

\section{RBDMFT equations}

In deriving the effective action, we consider the limit of a high but finite dimensional optical lattice, and use the cavity method $[1,2]$ to derive self-consistency equations within RBDMFT. In a more formal language, first we map the Hamiltonian onto a set of individual single-site problems each of which is described by a local effective action [3]:

$$
\begin{aligned}
S_{\mathrm{imp}}^{(i)}= & -\int_{0}^{\beta} d \tau d \tau^{\prime} \sum_{\sigma \sigma^{\prime}}\left(\begin{array}{ll}
b_{0, \sigma}^{(i)}(\tau)^{*} & \left.b_{0, \sigma}^{(i)}(\tau)\right)
\end{array}\right) \mathcal{G}_{0, \sigma \sigma^{\prime}}^{(i)}\left(\tau-\tau^{\prime}\right)^{-1}\left(\begin{array}{c}
b_{0, \sigma^{\prime}}^{(i)}\left(\tau^{\prime}\right) \\
b_{0, \sigma^{\prime}}^{(i)}\left(\tau^{\prime}\right)^{*}
\end{array}\right)+ \\
& \int_{0}^{\beta} d \tau\left\{\frac{1}{2} U_{\sigma \sigma^{\prime}} n_{0, \sigma}^{(i)}(\tau)\left(n_{0, \sigma^{\prime}}^{(i)}(\tau)-\delta_{\sigma \sigma^{\prime}}\right)+\sum_{j(j \neq 0)} V_{0 j} n_{0, d}^{(i)}(\tau) n_{j, d}^{(i)}(\tau)-\sum_{\langle 0 j\rangle, \sigma} t_{\sigma}\left(b_{0, \sigma}^{(i)}(\tau)^{*} \phi_{j, \sigma}^{(i)}(\tau)+b_{0, \sigma}^{(i)}(\tau) \phi_{j, \sigma}^{(i)}(\tau)^{*}\right)\right\} .
\end{aligned}
$$

Here we have defined the local Weiss Green's function,

$$
\mathcal{G}_{0, \sigma \sigma^{\prime}}^{-1}\left(\tau-\tau^{\prime}\right) \equiv-\left(\begin{array}{cc}
\left(\partial_{\tau^{\prime}}-\mu_{\sigma}\right) \delta_{\sigma \sigma^{\prime}}+t^{2} \sum G_{\sigma \sigma^{\prime}, i j}\left(\tau, \tau^{\prime}\right) & t^{2} \sum_{\langle 0 i\rangle,\langle 0 j\rangle} G_{\sigma \sigma^{\prime}, i j}^{2}\left(\tau, \tau^{\prime}\right) \\
t^{2} \sum_{\langle 0 i\rangle,\langle 0 j\rangle} G_{\sigma \sigma^{\prime}, i j}^{2}{ }^{*}\left(\tau^{\prime}, \tau\right) & \left(-\partial_{\tau^{\prime}}-\mu_{\sigma}\right) \delta_{\sigma \sigma^{\prime}}+t^{2} \sum_{\langle 0 i\rangle,\langle 0 j\rangle} G_{\sigma \sigma^{\prime}, i j}^{1}\left(\tau^{\prime}, \tau\right)
\end{array}\right),
$$

and introduced

$$
\phi_{i, \sigma}(\tau) \equiv\left\langle b_{i, \sigma}(\tau)\right\rangle_{0}
$$

as the superfluid order parameters, and

$$
\begin{aligned}
& G_{\sigma \sigma^{\prime}, i j}^{1}\left(\tau, \tau^{\prime}\right) \equiv-\left\langle b_{i, \sigma}(\tau) b_{j, \sigma^{\prime}}^{*}\left(\tau^{\prime}\right)\right\rangle_{0}+\phi_{i, \sigma^{\prime}}(\tau) \phi_{j, \sigma}^{*}\left(\tau^{\prime}\right), \\
& G_{\sigma \sigma^{\prime}, i j}^{2}\left(\tau, \tau^{\prime}\right) \equiv-\left\langle b_{i, \sigma}(\tau) b_{j, \sigma^{\prime}}\left(\tau^{\prime}\right)\right\rangle_{0}+\phi_{i, \sigma^{\prime}}(\tau) \phi_{j, \sigma}\left(\tau^{\prime}\right)
\end{aligned}
$$


as the diagonal and off-diagonal parts of the connected Green's functions, respectively, where $\langle\ldots\rangle_{0}$ denotes the expectation value in the cavity system (without the impurity site) [3, 4].

\section{Anderson impurity model}

The most difficult step in the procedure discussed above is to find a solver for the effective action. Howev- er, one cannot do this analytically. To obtain RBDMFT equations, it is better to return back to the Hamiltonian representation. Here, each of the local effective actions (A1) is represented by an Anderson impurity Hamiltonian

$$
\begin{aligned}
\hat{H}_{A}= & -\sum_{\langle 0 j\rangle \sigma} t_{\sigma}\left(\phi_{j, \sigma}^{*} \hat{b}_{0, \sigma}+\text { h.c. }\right)+\frac{1}{2} \sum_{\sigma \sigma^{\prime}} U_{\sigma \sigma^{\prime}} \hat{n}_{0, \sigma}\left(\hat{n}_{0, \sigma^{\prime}}-\delta_{\sigma \sigma^{\prime}}\right)+\sum_{j(j \neq 0)} V_{j 0}\left\langle\hat{n}_{j, d}\right\rangle \hat{n}_{0, d}-\sum_{\sigma} \mu_{0, \sigma} \hat{n}_{0, \sigma} \\
& +\sum_{l} \epsilon_{l} \hat{a}_{l}^{\dagger} \hat{a}_{l}+\sum_{l, \sigma}\left(V_{\sigma, l} \hat{a}_{l}^{\dagger} \hat{b}_{0, \sigma}+W_{\sigma, l} \hat{a}_{l} \hat{b}_{0, \sigma}+\text { h.c. }\right)
\end{aligned}
$$

where the chemical potential and interaction term are directly inherited from the Hubbard Hamiltonian. The bath of condensed bosons is represented by the Gutzwiller term with superfluid order parameters $\phi_{\sigma}$ for each component. The bath of normal bosons is described by a finite number of orbitals with creation operators $\hat{a}_{l}^{\dagger}$ and energies $\epsilon_{l}$, where these orbitals are coupled to the impurity via normal-hopping amplitudes $V_{\sigma, l}$ and anomalous-hopping amplitudes $W_{\sigma, l}$. The anomalous hopping terms are needed to generate the off-diagonal elements of the hybridization function. Note here that in the high-dimensional limit inter-site interactions only contribute to the Hartree level [5]. In other words, the Hartree term of the inter-site interaction will dominate as the spatial dimension of the system increases.
This motivates us to keep only the Hartree contribution of the inter-site interaction in our simulations as an approximation to the original Hamiltonian, i.e.

$$
\frac{1}{2} \sum_{i \neq j} V_{i j} \hat{n}_{i, d} \hat{n}_{j, d} \approx \sum_{i \neq j} V_{i j}\left\langle\hat{n}_{i, d}\right\rangle\left(\hat{n}_{j, d}-\frac{1}{2}\left\langle\hat{n}_{i, d}\right\rangle\right)
$$

We now turn to the solution of the impurity model. In practice, we start with an initial set of Anderson paramters and local bosonic superfluid order parameters $\phi_{j, \nu}(\tau)$. The Anderson Hamiltonian can straightforwardly be implemented in the Fock basis, and the corresponding solution can be achieved by exact diagonalization (ED) of DMFT [1, 6]. After diagonalization, the local Green's function, which includes all the information about the bath, can be obtained from the eigenstates and eigenenergies in the Lehmann-representation

$$
\begin{aligned}
& G_{\mathrm{imp}, \sigma \sigma^{\prime}}^{1}\left(i \omega_{n}\right)=\frac{1}{Z} \sum_{m n}\left\langle m\left|\hat{b}_{\sigma}\right| n\right\rangle\left\langle n\left|\hat{b}_{\sigma^{\prime}}^{\dagger}\right| m\right\rangle \frac{e^{-\beta E_{n}}-e^{-\beta E_{m}}}{E_{n}-E_{m}+i \hbar \omega_{n}}+\beta \phi_{\sigma} \phi_{\sigma^{\prime}}^{*} \\
& G_{\mathrm{imp}, \sigma \sigma^{\prime}}^{2}\left(i \omega_{n}\right)=\frac{1}{Z} \sum_{m n}\left\langle m\left|\hat{b}_{\sigma}\right| n\right\rangle\left\langle n\left|\hat{b}_{\sigma^{\prime}}\right| m\right\rangle \frac{e^{-\beta E_{n}}-e^{-\beta E_{m}}}{E_{n}-E_{m}+i \hbar \omega_{n}}+\beta \phi_{\sigma} \phi_{\sigma^{\prime}}
\end{aligned}
$$

Integrating out the orbitals leads to the same effective action as in Eq. (A1), if the following identification is made

$$
\boldsymbol{\Delta}_{\sigma \sigma^{\prime}}\left(i \omega_{n}\right) \equiv t^{2} \sum_{\langle 0 i\rangle,\langle 0 j\rangle} \mathbf{G}_{\sigma \sigma^{\prime}, i j}\left(i \omega_{n}\right)
$$

where $\mathbf{G}_{\sigma \sigma^{\prime}, i j}\left(i \omega_{n}\right)$ is the inverse Fourier transformation of the Weiss Green's function defined in Eq. (4) and (5), and the hybridization functions read:

$$
\begin{aligned}
& \Delta_{\sigma \sigma^{\prime}}^{1}\left(i \omega_{n}\right) \equiv \sum_{l}\left(\frac{V_{\sigma, l} V_{\sigma^{\prime}, l}}{\epsilon_{l}-i \omega_{n}}+\frac{W_{\sigma, l} W_{\sigma^{\prime}, l}}{\epsilon_{l}+i \omega_{n}}\right) \\
& \Delta_{\sigma \sigma^{\prime}}^{2}\left(i \omega_{n}\right) \equiv \sum_{l}\left(\frac{V_{\sigma, l} W_{\sigma^{\prime}, l}}{\epsilon_{l}-i \omega_{n}}+\frac{W_{\sigma, l} V_{\sigma^{\prime}, l}}{\epsilon_{l}+i \omega_{n}}\right) .
\end{aligned}
$$


Hence, we obtain a set of local self-energies $\Sigma_{\mathrm{imp}, \sigma \sigma^{\prime}}^{(i)}\left(i \omega_{n}\right)$,

$$
\begin{aligned}
\boldsymbol{\Sigma}_{\mathrm{imp}, \sigma \sigma^{\prime}}\left(i \omega_{n}\right) & =\left(i \omega_{n} \sigma_{z}+\mu_{\sigma}\right) \delta_{\sigma \sigma^{\prime}} \\
& +\boldsymbol{\Delta}_{\sigma \sigma^{\prime}}\left(i \omega_{n}\right)-\mathbf{G}_{\mathrm{imp}, \sigma \sigma^{\prime}}^{-1}\left(i \omega_{n}\right) .
\end{aligned}
$$

Then we employ the Dyson equation in real-space representation in order to compute the interacting lattice Green's function

$$
\mathbf{G}\left(i \omega_{n}\right)^{-1}=\mathbf{G}_{0}\left(i \omega_{n}\right)^{-1}-\boldsymbol{\Sigma}\left(i \omega_{n}\right) .
$$

The site-dependence of the Green's functions is shown by boldface quantities that denote a matrix form with siteindexed elements. Here $\mathbf{G}_{0}\left(i \omega_{n}\right)^{-1}$ stands for the inverse non-interacting Green's function

$$
\mathbf{G}_{0}\left(i \omega_{n}\right)^{-1}=\left(\mu+i \omega_{n}\right) \mathbf{1}-\mathbf{t} .
$$

In this expression, $\mathbf{1}$ is the unit matrix, the matrix elements $t_{i j}$ are hopping amplitudes for a given lattice structure. Eventually the self-consistency loop is closed by specifying the Weiss Green's function via the local Dyson equation

$$
\left(\mathcal{G}_{0, \sigma \sigma^{\prime}}^{(i)}\left(i \omega_{n}\right)\right)^{-1}=\left(\mathbf{G}_{\sigma \sigma^{\prime}}^{(i)}\left(i \omega_{n}\right)\right)^{-1}+\boldsymbol{\Sigma}_{\sigma \sigma^{\prime}}^{(i)}\left(i \omega_{n}\right),
$$

where the diagonal elements of the lattice Green's function yield the interacting local Green's function $\mathbf{G}_{\sigma \sigma^{\prime}}^{(i)}\left(i \omega_{n}\right)=\left(\mathbf{G}_{\sigma, \sigma^{\prime}}\left(i \omega_{n}\right)\right)_{i i}$. This self-consistency loop is repeated until the desired accuracy for superfluid order parameters and Anderson parameters is obtained.

\section{Energy within RBDMFT}

Calculation of energy is not straightforward within RBDMFT, since the kinetic energy kinetic is given in terms of non-local expectation values. It can be shown that within the RBDMFT self-consistency conditions, kinetic energy can also be written in terms of Anderson impurity hybridization functions and local Green's functions. A detailed derivation can be found in Ref. [7].

\section{a. Kinetic energy}

In terms of creation and annihilation operators for bosons, $b_{i \sigma}^{\dagger}$ and $b_{i \sigma}$, respectively, kinetic energy has the form

$$
\hat{H}_{\mathrm{kin}}=-\sum_{\langle i j\rangle, \sigma} t_{\sigma}\left(b_{i, \sigma}^{\dagger} b_{j, \sigma}+\text { H.c. }\right) .
$$

Thus expressing the total kinetic energy in terms of real-space Green's functions yields

$$
\begin{aligned}
E_{\mathrm{kin}} & =-\sum_{i j, \sigma} t_{i j}^{\sigma}\left\langle\hat{b}_{\sigma, i}^{\dagger} \hat{b}_{\sigma, j}\right\rangle \\
& =\sum_{i j, \sigma} t_{i j}^{\sigma}\left(\lim _{\epsilon \rightarrow 0^{+}} \sum_{n=-\infty}^{\infty} \frac{e^{i \omega_{n} \epsilon}}{\beta} G_{j i, \sigma}\left(i \omega_{n}\right)-\phi_{i, \sigma}^{*} \phi_{j, \sigma}\right)
\end{aligned}
$$

This expression can be further simplified by employing both the local and lattice Dyson equations within RBDMFT

$$
\mathbf{G}_{i}^{C}\left(i \omega_{n}\right)^{-1}=i \omega_{n} \sigma_{z}+\mu+\boldsymbol{\Delta}_{i}\left(i \omega_{n}\right)-\boldsymbol{\Sigma}_{i}\left(i \omega_{n}\right)
$$

$$
\left[\mathbf{G}_{R}^{C}\left(i \omega_{n}\right)^{-1}\right]_{i j}=t_{i j} \mathbf{1}+\delta_{i j}\left(i \omega_{n} \sigma_{z}+\mu \mathbf{1}-\boldsymbol{\Sigma}_{i}\left(i \omega_{n}\right)\right),
$$

which yields

$$
\sum_{j}\left[\mathbf{G}_{R}^{C}\left(i \omega_{n}\right)^{-1}\right]_{i j}\left[\mathbf{G}\left(i \omega_{n}\right)\right]_{j i}=\sum_{j}\left[t_{i j} \mathbf{1}_{2}-\delta_{i j}\left(\boldsymbol{\Delta}_{i}\left(i \omega_{n}\right)-\mathbf{G}_{i}\left(i \omega_{n}\right)^{-1}\right)\right]\left[\mathbf{G}\left(i \omega_{n}\right)\right]_{j i}
$$

Further using the self-consistency property of the impurity Green's function leads to

$$
\sum_{j} t_{i j}\left[\mathbf{G}\left(i \omega_{n}\right)\right]_{j i}=\boldsymbol{\Delta}_{i}\left(i \omega_{n}\right) \mathbf{G}_{i}\left(i \omega_{n}\right) .
$$

and we finally obtain

$$
\begin{aligned}
E_{\mathrm{kin}}= & \frac{2}{\beta} \lim _{\epsilon \rightarrow 0^{+}} \sum_{i \sigma n \geq 0} \operatorname{Re}\left(\left[\boldsymbol{\Delta}_{\sigma, i}\left(i \omega_{n}\right) \mathbf{G}_{\sigma, i}\left(i \omega_{n}\right)\right]_{11} e^{i \omega_{n} \epsilon}\right) \\
& -\sum_{i j \sigma} t_{i j} \phi_{i, \sigma}^{*} \phi_{j, \sigma}-\frac{\operatorname{Tr}\left[\boldsymbol{\Delta}_{\sigma, i}(0) \mathbf{G}_{\sigma, i}(0)\right]}{2 \beta} . \quad \text { (A23) }
\end{aligned}
$$

\section{b. Total energy}

The ground state within RBDMFT corresponds to the solution with the lowest energy, where the corresponding total energy of the impurity site which is given as follows:

$$
E=E_{\text {kin }}+E_{\text {int }} .
$$

For the Bose-Hubbard model of spin-1 bosons, the on-site interaction term is given by:

$$
E_{\mathrm{int}}=\frac{1}{2} \sum_{i, \sigma \sigma^{\prime}} U_{\sigma \sigma^{\prime}} \hat{n}_{i, \sigma}\left(\hat{n}_{i, \sigma^{\prime}}-\delta_{\sigma \sigma^{\prime}}\right)+\sum_{i<j} V_{i j} \hat{n}_{i, d} \hat{n}_{j, d}
$$


Appendix B: Numerical results of the dipolar system

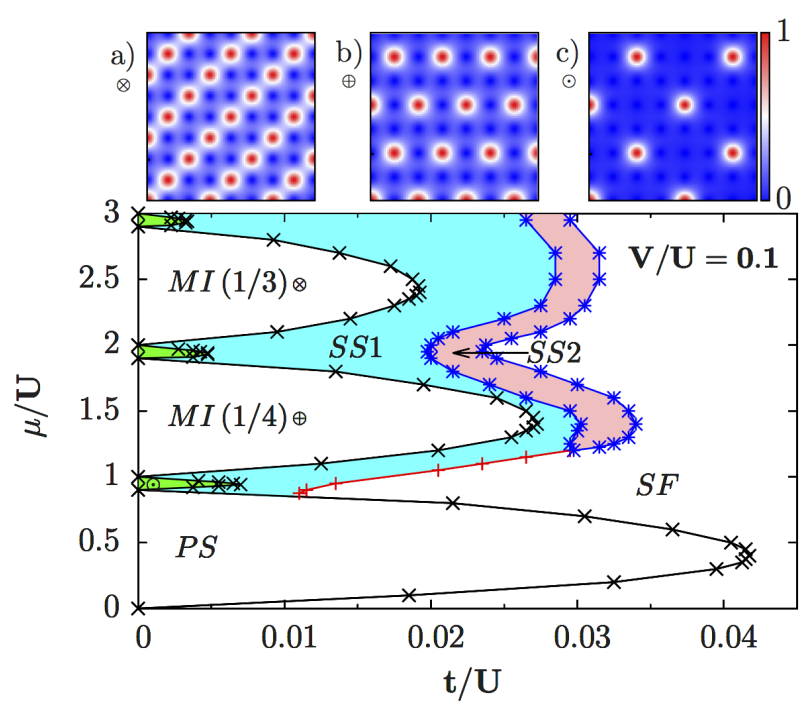

FIG. 7. (Color online) Phase diagram for a mixture of nondipolar species $b$ and dipolar component $d$ on a square lattice for a dipolar interaction strength $V / U=0.1$, exhibiting pronounced regions of supersolid marked by the cyan (SS1) and pink color (SS2). In contrast to nearest-neighbor case [11], the system demonstrates various crystalline order, as shown in a)-c) for the real-space density distribution of the dipolar species. Note here that, in the DW, marked by the green color, the total density distributes spatially nonuniform with a homogeneous density for the nondipolar species, whereas, in the MI, the total density distribute spatially uniform. We observe a phase separation (PS) in the MI region with a total filling $n_{b}+n_{d}=1$, in addition to spatially uniform superfluid (SF). Other parameters are $U_{b d}=0.9 U$, and $\mu_{b, d}=\mu$.

We have so far studied crystalline order in the Rydberg dressed systems. Actually, the physics of these competing orders can also be exhibited in dipolar system loaded in an optical lattice, along with quick developments in the cooling and trapping of magnetic atoms [9] and diatomic molecules [10]. Recently, a Gutzwiller mean-field phase diagram of a binary Bose mixture on a square optical lattice is studied, where one species possesses a nonnegligible dipole moment [11]. In their study, only the nearest-neighbor part of the dipolar interactions was included. To obtain a better understanding of the Rydberg dressed system studied above and make a comparison, we here study a mixture of dipolar and nondipolar bosons on a square optical lattice, with real long-range interactions beyond nearest-neighbor approximations. We study the system by means of RBDMFT, which takes into account quantum fluctuations and is actually a higher-order expansions of Gutzwiller mean-field theory.

In Fig. 7, we show the resulting phase diagram of dipolar and nondipolar bosonic mixtures on a 2D optical lattice. In general, there are also five phases in this dipolar system, i.e. SF, MI, DW and two types of supersol- id. Compared to nearest-neighbor interaction and static mean-field approximations [11], two big differences have been observed. First, rich crystalline patterns appear in the system, as shown in Fig. 7a)-c), with a filling factor of $1 / 3,1 / 4$ and $1 / 8$ for the dipolar species, respectively. Second, we observe that the region of supersolid phase is also altered. Note here that we recover the static meanfield phase diagram with nearest-neighbor interactions within Gutzwiller approximations in Ref. [11].

\section{Appendix C: Bogoliubov spectra of the bare species in the SS1 phase}

Using Fourier transformation, Hamiltonian $\hat{H}_{\mathbf{e}}$ is changed to the momentum space,

$$
\begin{gathered}
\tilde{H}=-\sum_{\vec{k}}\left[\mu+2 t\left(\cos k_{x} a+\cos k_{y} a\right)\right] b_{\vec{k}}^{\dagger} b_{\vec{k}}+ \\
\frac{U}{2 N} \sum_{\vec{k}_{1} \vec{k}_{2} \vec{k}_{3}} b_{\vec{k}_{1}}^{\dagger} b_{\vec{k}_{3}}^{\dagger} b_{\vec{k}_{3}+\vec{k}_{2}} b_{\vec{k}_{1}-\vec{k}_{2}}+U_{1} \sum_{\{\vec{k}\}} b_{\vec{k}}^{\dagger} b_{\vec{k}},
\end{gathered}
$$

where $N$ is the total number of sites and $U_{1}=\bar{n}_{d} U_{b d}$ with $\bar{n}_{d}=n_{d} N_{d} / N . N_{d}$ is the number of sites occupied by the dressed atoms, and $\{k\}$ denotes momentum spanned in the first Brillouin zone of the lattice occupied by the dressed atoms.

Expanding the Hamiltonian (C1) around $|\vec{k}|=0$ and keeping only quadratic terms of the operators, this yields,

$$
\begin{aligned}
\tilde{H}_{\mathrm{B}} \approx-\sum_{\vec{k} \neq 0}\left[\mu+2 t\left(\cos k_{x} a+\cos k_{y} a\right)-2 U \bar{n}_{b}\right] b_{\vec{k}}^{\dagger} b_{\vec{k}} \\
+\frac{U \bar{n}_{b}}{2} \sum_{\vec{k} \neq 0}\left(b_{\vec{k}} b_{-\vec{k}}+b_{-\vec{k}}^{\dagger} b_{\vec{k}}^{\dagger}\right)+U_{1} \sum_{\{\vec{k} \neq 0\}} b_{\vec{k}}^{\dagger} b_{\vec{k}},
\end{aligned}
$$

where $\tilde{H}_{\mathrm{B}}=\tilde{H}-E_{0}$ is the Hamiltonian of the phonons and $E_{0}=-U N_{0}^{2} / 2 N$ is the energy of the condensed atoms, with $N_{0}$ to be the number of condensed atoms and $\mu=-4 t+U \bar{n}_{b}+U_{1}$ the chemical potential and the mean occupation of the condensed atom $\bar{n}_{b}=N_{0} / N$.

As the interspecies interaction the last term in $\mathrm{E}$ q. (C2)] only appears in the low momentum regions (Brillouin zone $\{k\}$ ), we will have two different forms of the approximate Hamiltonian depending on values of the momentum. Substituting the chemical potential $\mu$, we get the approximate Hamiltonian within the first Brillouin zone of the dressed atom,

$$
\tilde{H}_{\mathrm{B}} \approx \sum_{\vec{k} \neq 0}\left[\varepsilon_{k}+U \bar{n}_{b}\right] b_{\vec{k}}^{\dagger} b_{\vec{k}}+\frac{U \bar{n}_{b}}{2} \sum_{\vec{k} \neq 0}\left(b_{\vec{k}} b_{-\vec{k}}+b_{-\vec{k}}^{\dagger} b_{\vec{k}}^{\dagger}\right),
$$

and the corresponding Bogoliubov spectrum is

$$
E_{l}(k)=\sqrt{\varepsilon_{k}\left(\varepsilon_{k}+2 U \bar{n}_{b}\right)},
$$

with $\varepsilon_{k}=-2 t\left(\cos k_{x} a+\cos k_{y} a-2\right)$. The spectrum is similar to the one of a weakly interacting Bose gas in a square optical lattices. 
For momenta outside the first Brillouin zone of the dressed atoms, we have a different form of the approximate Hamiltonian,

$\tilde{H}_{\mathrm{B}} \approx \sum_{\vec{k} \neq 0}\left[\varepsilon_{k}+U \bar{n}_{b}-U_{1}\right] b_{\vec{k}}^{\dagger} b_{\vec{k}}+\frac{U \bar{n}_{b}}{2} \sum_{\vec{k} \neq 0}\left(b_{\vec{k}} b_{-\vec{k}}+\right.$ H.c. $)$,

the corresponding Bogoliubov spectra is

$$
E_{l}(k)=\sqrt{\left(\varepsilon_{k}-U_{1}\right)\left(\varepsilon_{k}-U_{1}+2 U \bar{n}_{b}\right)},
$$

which will be nonzero only at large momentum (outside the first Brillouin zone).

The roton instability occurs at the boundary of the two Bogoliubov spectrum. Using Eq. (C4), we can find the spectrum becomes complex when $\varepsilon_{k}<U_{1}$. This allows us to find the critical value of the tunneling rate $t_{c}$

$$
t_{c}=\frac{U_{1}}{2\left[2-\cos k_{x}^{(b)}-\cos k_{y}^{(b)}\right]},
$$

where $k_{x}^{(b)}$ and $k_{y}^{(b)}$ are values of the momentum at the boundary of the first Brillouin zone of the oblique lattice.

The soft-core interaction will affect structures of the oblique lattice. Therefore the critical $t_{c}$ will change as the interaction $V$ changes. As shown in Fig. 3 in the main text, the first Brillouin zone is not of a regular shape, such that the critical value $t_{c}$ will vary with both $k_{x}^{(b)}$ and $k_{y}^{(b)}$. To show this we evaluate the critical values $t_{c}$ using the crystalline structure of the dressed atoms at the SS1-SS2 phase boundary, which are obtained by the full numerical calculation. For example, $t_{c}$ lies in a range $[0.087,0.094]$ if $V=0.3$. When the long range interaction becomes strong, we find that the range of critical $t_{c}$ increases. For example, $t_{c} \in[0.085,0.11]$ when $V=0.4$, and $t_{c} \in[0.073,0.13]$ when $V=0.6$. Although these values are close to the numerical calculations, it is apparent that one will not be able to determine phase boundaries accurately using the Bogoliubov calculation.

Another limitation of this calculation is that areas of the crystalline structure become smaller when $V$ is weak. Long range correlations become important in determining the ground state phases, which prevents us to decouple the total wave function into two parts. In this regime, the Bogoliubov calculation fails to capture the many body physics.

[1] A. Georges, G. Kotliar, W. Krauth and M. J. Rozenberg, Rev. Mod. Phys. 68, 13 (1996).

[2] K. Byczuk and D. Vollhardt, Phys. Rev. B 77, 235106 (2008).

[3] Y.-Q. Li et al., Phys. Rev. B 84, 144411 (2011).

[4] M. Snoek and W. Hofstetter, Bosonic Dynamical Mean-Field Theory Chapter in "Quantum Gases: Finite Temperature and Non-Equilibrium Dynamics", N.P. Proukakis et al., (Imperial College Press, London, 2013).

[5] E. Müller-Hartmann, Z. Phys. B 74, 507 (1989).

[6] M. Caffarel and W. Krauth, Phys. Rev. Lett. 72, 1545 (1994).

[7] Andreas Geißler et al., Phys. Rev. A 95, 063608 (2017)..

[8] G. Pupillo, A. Michelim, M. Boninsegni, I. Lesanovsky and P. Zoller, Phys. Rev. Lett. 104, 223002 (2010).

[9] A. Griesma et al., Phys. Rev. Lett. 94, 160401 (2005); M. Lu et al., Phys. Rev. Lett. 107, 190401 (2011); K. Aikawa et al., Phys. Rev. Lett. 108, 210401 (2012); S. Baier et al., Science 352, 201 (2016).

[10] K.-K. Ni et al., Science 322, 231 (2008); S. A. Moses et al., Nat. (London) 350, 656 (2015); J. Deiglmayr et al., Phys. Rev. Lett 101, 133004 (2008); T. Takekoshi et al., Phys. Rev. Lett. 113, 205301 (2014); P. K. Molony et al., Phys. Rev. Lett. 113, 255301 (2014); J. W. Park et al., Phys. Rev. Lett. 114, 205302 (2015).

[11] R. M. Wilson, W. E. Shirley, and S. S. Natu, Phys. Rev. A 93, 011605(R) (2016). 